\title{
The Miami International Evidence-Based Guidelines on Minimally Invasive Pancreas Resection: Moving from Initial Adoption to Thoughtful Dissemination
}

\author{
Daniel A. Anaya, $\mathrm{MD}^{1}$, Ugwuji Maduekwe, $\mathrm{MD}^{2}$, and Jin $\mathrm{He}, \mathrm{MD}, \mathrm{PhD}^{3}$ \\ ${ }^{1}$ Section of Hepatobiliary Tumors, Department of Gastrointestinal Oncology, H. Lee Moffitt Cancer Center and Research \\ Institute, Tampa, FL; ${ }^{2}$ Division of Surgical Oncology and Endocrine Surgery, Department of Surgery, University of North \\ Carolina School of Medicine, Chapel Hill, NC; ${ }^{3}$ Department of Surgery, Johns Hopkins University School of Medicine, \\ Baltimore, MD
}

\section{BACKGROUND INFORMATION}

The International Consensus Conference on Minimally Invasive Pancreatic Surgery to review and vote on a final draft of guidelines on minimally invasive pancreas resection (MIPR) took place on 18-19 March 2019 in Miami. The Society of Surgical Oncology (SSO) provided sponsorship support of the meeting. The recent publication of the Miami International Evidence-Based Guidelines on Minimally Invasive Pancreas Resection indicated SSO endorsement of the guidelines. The MIPR guidelines were reviewed by three members of the SSO Hepato-PancreatoBiliary Disease Site Working Group and three members of the SSO Quality Committee. Reviewers agreed the MIPR guidelines were well developed through the use of a validated methodology, the involvement of multiple stakeholders, and critical review by an audience of surgeons with an interest in pancreatic cancer. Although there are limitations in the data available to support the MIPR guidelines, reviewers felt this was acknowledged by authors, and their 'call' to the profession is to do better. Additionally, reviewers felt the potential impact of the guidelines is significant with respect to the creation of a

Daniel A. Anaya, Ugwuji Maduekwe, and Jin He are members of the Society of Surgical Oncology HPB Disease Site Working Group.

(C) Society of Surgical Oncology 2020

First Received: 22 January 2020;

Published Online: 17 March 2020

D. A. Anaya, MD

e-mail: daniel.anaya@moffitt.org framework to help guide the advancement and dissemination of novel surgical techniques without compromising the provision of safe, high-quality care. Based on the reviewer's feedback and recommendations, the SSO leadership agreed that the Society endorse the guidelines.

The Miami International Evidence-Based Guidelines on Minimally Invasive Pancreas Resection were recently published in Annals of Surgery. ${ }^{1}$ The manuscript summarized work developed by an international collaboration of experts and stakeholders in the field of minimally invasive pancreatic resection (MIPR) from across 20 countries and included results leading to and derived from the International Guidelines MIPR meeting held in Miami on 18-19 March 2019. This project built upon previous experience from the first International state-of-the-art meeting on MIPR (Sao Paulo, 2016), and focused on 'areas of controversy', including six distinct topics: Distal and Central Pancreatectomy, Pancreatoduodenectomy, Patients and Technique, Training and Implementation, Instrumentation, and Accountability. The authors ought to be congratulated for this work as it addresses critical areas in the rapidly evolving field of MIPR by presenting the current landscape and evidence, and framing the practice of MIPR (current and future) in the context of safety and efficiency. Most notably, these guidelines represent an example of true academic and professional collaboration led within the most strict and rigorous methodology, including creation of task-based and topic-specific committees, a priori assignment of every participant's role, an iterative Delphi process for the development and evaluation of each question along with recommendations, and a number of methodologic standards (i.e. Scottish Intercollegiate Guidelines Network [SIGN], which include using GRADE for quality/level of 
evidence and strength of recommendations, and AGREE II to assess the quality of the guidelines) ${ }^{2}$ for development and validation of the guidelines. As such, the final report provides well-informed recommendations, with measures of quality, strength and validity, as well as additional remarks and proposed actions for each of the questions addressed, making these guidelines a valuable tool for the reader when considering MIPR practices.

The critical assessment of reviewed data brings to light advances that have occurred in the field of MIPR during the last decade, and the basis for the focus on evolving controversies within the practice of MIPR. As with other innovations, after adoption occurs the challenge is balancing dissemination with fidelity during implementation of such practice. ${ }^{3}$ These guidelines provide standards for safety and efficiency of MIPR in selected topics, serving as a framework for current practice and further dissemination. The participation of only clinical experts during guideline development has both advantages and disadvantages, and could be interpreted as a seemingly obvious limitation, particularly when considering that MIPR is also occurring in smaller academic practices and community-based centers. ${ }^{4,5}$ However, the authors overcome this limitation with the breadth and depth of the international consensus, including experts from 20 countries representing different patient populations and practice characteristics. Similarly, MIPR encompasses different highly complex operations with data supporting a selective approach when considering dissemination outside of specialized high-volume centers and by surgeons with unique required skills. ${ }^{6,7}$ Understanding these requirements, limitations, and ideal context for these highest-tier complex procedures (i.e. pancreatoduodenectomy and vascular resection/reconstruction) is essential to maintain appropriate safety and efficiency standards. Leveraging the participation of experts in this narrow field and using objective methods to define the degree to which a widespread dissemination can and/or should be promoted is critical for patient care and to the field, and can have important public health implications (population-level). These guidelines serve as a first step to define and frame these topics. While the surgical community should welcome these efforts, it also looks forward to having collaborative groups such as this one take-on the challenges highlighted in the manuscript so as to continue advancing the field using the most rigorous methodologies.

Among the different questions addressed in these guidelines, a strong and high-quality-level recommendation was made for the use of MIPR in distal pancreatectomy over open resection for benign and lowgrade tumors. The benefits derived from this approach include a shorter length of stay (LOS) and reduced blood loss, while overall complication rates are equivalent. These findings are important as they support a shift for the use of distal pancreatectomy for these indications to a minimally invasive approach. Nonetheless, a number of other important issues are considered when planning for an operation, beyond indications. A patient's comorbidities and tumor features are consistently used by surgeons to help strategize a surgical approach, and more so when performing complex operations. ${ }^{4}$ Although the guidelines do not denote any contraindications for MIPR based on age, obesity, and/or prior abdominal surgeries, other important features such as prior pancreatitis or tumor size and relations to other structures were not specifically addressed. This is a shortcoming in part due to the scope of the guidelines; nevertheless, it is important to emphasize that patient selection in the context of these factors varies, and, as the authors point out, multi-institutional efforts should focus on prospective collection of data addressing these factors.

At least four of the six topics addressed dealt with other clinical care questions, including specific surgical procedures and technique. A common theme was the lack of high-level prospective data to support a given practice. This was especially true for MIPR for vascular resection/ reconstruction, cost, quality-of-life outcomes, and a number of technique- and device/instrumentation-related topics. It is noteworthy that the authors reported there was no data or guidance regarding the best approach for bleeding control during surgery. In the context of surgical innovation, mirroring approaches used for the development and testing of a new drug, safety concerns lead the first steps when introducing a new approach (e.g. phase I trials). These findings are critical; as dissemination of MIPR expands outside of highly specialized centers, strategies for surgical rescue from a bleeding event (and others) must be developed and clearly outlined to maintain the safety standards guiding surgical practice. ${ }^{8}$ Similarly, the authors report equivalent outcomes observed using MIPR for adenocarcinoma, although appropriately acknowledge the need for additional prospective data supporting the use of MIPR for pancreatic adenocarcinoma and for MIPR in the context of neoadjuvant approaches. One should not forget lessons learned from other innovation processes in cancer, including the use of laparoscopic resections for rectal cancer, in which, despite the appearance of equivalent short-term and oncologic outcomes, the initial randomized trials failed to prove a non-inferiority effect of the laparoscopic approach versus the open approach. ${ }^{9,10} \mathrm{Cer}-$ tainly, results from ongoing trials comparing MIPR and open approaches in patients with adenocarcinoma, and evaluation of the impact on neoadjuvant 
chemotherapy \pm radiation on short-term and oncologic outcomes, will better inform these questions and the indication, advantages, and/or disadvantages of MIPR for cancer.

Pivotal contributions of these guidelines include those related to topics addressed beyond clinical care, specifically regarding implementation, training, and accountability. As previously described, there is a clear correlation between hospital and surgeon's case volume and outcome for complex pancreatic resections, ${ }^{11-13}$ and this association also holds true for MIPR. ${ }^{14}$ Strong recommendations were made for performing MIPRpancreatoduodenectomy (MIPD) at high-volume centers, with $>20 \mathrm{MIPDs} /$ year used as the recommended threshold (associated with lower complications). Equally relevant was the data emphasizing the importance of training and prolonged learning curves. Although it is difficult to account for inter-surgeon and practice variation to define a threshold of cases to be used as a benchmark, the guidelines do an excellent job in framing the context for training, including timing (early on), skills required, and models of structured training programs. ${ }^{15-17}$ One of the most important recommendations, common to the multiple questions addressed, is for mandatory participation in national/international registries in MIPR. The authors should be commended for pushing this practice and for starting the conversation regarding more formal recognition (perhaps accreditation for MIPR through surgical societies or other mechanisms) using safety and efficiency standards and including program structure, training processes, and outcomes data.

In summary, the Miami International Evidence-Based Guidelines on Minimally Invasive Pancreas Resection have been developed within the highest methodologic standards and address key controversial issues regarding the practice, implementation, and dissemination of MIPR presently and for the future. These guidelines should become the reference work to inform and guide surgeons and hospitals regarding the practice of MIPR. Importantly, they lay out action items to further this work, including the establishment of multi-institutional registries focused on prospective evaluation of key topics in MIPR, as well as training and implementation of MIPR within the standards outlined by the guidelines. The challenges ahead include collaborative work to develop a framework promoting (enforcing) participation in such registries, creating the appropriate setting for accreditation of hospitals and surgeons, and the execution of multi-institutional clinical trials to fill the clinical gaps outlined in the manuscript, with a special focus on those related to MIPR and multidisciplinary pancreatic cancer care. As these are developed, important questions will have to be addressed related to the ideal settings for MIPR, the balance between widespread dissemination versus regionalization for the delivery of MIPR, and, in this context, the expectation and goals for trainees in the field of pancreatic surgery and MIPR. There is no doubt that collaborative work of this magnitude and rigor will help move these topics ahead, and should be encouraged as a means to advance the field while maintaining the central purpose of our practice-the patientsat the forefront of any innovation.

FUNDING Not applicable.

DISCLOSURE Daniel A. Anaya, Ugwuji Maduekwe, and Jin He declare no competing interests.

\section{REFERENCES}

1. Asbun HJ, Moekotte AL, Vissers FL, et al. The Miami international evidence-based guidelines on minimally invasive pancreas resection. Ann Surg. 2020;271(1):1-14.

2. Scottish Intercollegiate Guidelines Network (SIGN). SIGN 50: A guideline developer's handbook. SIGN publication no. 50 ed. Edinburgh: SIGN; 2019. https://www.sign.ac.uk/sign-50.html. Accessed 1 Dec 2019.

3. Glasglow R, Boles S, Vogt T. RE-AIM. http://re-aim.org. Accessed 23 Nov 2019.

4. Klompmaker S, van Zoggel DM, Watkins AA, et al. Nationwide evaluation of patient selection for minimally invasive distal pancreatectomy using American College of Surgeons' National Quality Improvement Program. Ann Surg. 2017;266(6): 1055-1061.

5. Ellis RJ, Zhang LM, Ko CY, et al. Variation in hospital utilization of minimally invasive distal pancreatectomy for localized pancreatic neoplasms. J Gastrointest Surg. Epub 25 Nov 2019.

6. van Hilst J, de Rooij T, Bosscha K, et al. Laparoscopic versus open pancreatoduodenectomy for pancreatic or periampullary tumours (LEOPARD-2): a multicentre, patient-blinded, randomised controlled phase 2/3 trial. Lancet Gastroenterol Hepatol. 2019;4(3):199-207.

7. Torphy RJ, Friedman C, Halpern A, et al. Comparing short-term and oncologic outcomes of minimally invasive versus open pancreaticoduodenectomy across low and high volume centers. Ann Surg. 2019;270(6):1147-1155.

8. King JC, Zeh HJ 3rd, Zureikat AH, et al. Safety in numbers: progressive implementation of a robotics program in an academic surgical oncology practice. Surg Innov. 2016;23(4): 407-414.

9. Stevenson AR, Solomon MJ, Lumley JW, et al. Effect of laparoscopic-assisted resection vs open resection on pathological outcomes in rectal cancer: the ALaCaRT randomized clinical trial. JAMA. 2015;314(13):1356-1363.

10. Fleshman J, Branda M, Sargent DJ, et al. Effect of laparoscopicassisted resection vs open resection of stage II or III rectal cancer on pathologic outcomes: the ACOSOG Z6051 randomized clinical trial. JAMA. 2015;314(13):1346-1355.

11. Birkmeyer JD, Siewers AE, Finlayson EV, et al. Hospital volume and surgical mortality in the United States. $N$ Engl J Med. 2002;346(15):1128-1137.

12. Birkmeyer JD, Stukel TA, Siewers AE, Goodney PP, Wennberg DE, Lucas FL. Surgeon volume and operative mortality in the United States. N Engl J Med. 2003;349(22):2117-2127. 
13. Hata T, Motoi F, Ishida M, et al. Effect of hospital volume on surgical outcomes after pancreaticoduodenectomy: a systematic review and meta-analysis. Ann Surg. 2016;263(4):664-672.

14. Moekotte AL, Rawashdeh A, Asbun HJ, et al. Safe implementation of minimally invasive pancreas resection: a systematic review. HPB (Oxford). Epub 10 Dec 2019.

15. Hogg ME, Besselink MG, Clavien PA, et al. Training in Minimally invasive pancreatic resections: a paradigm shift away from "See one, Do one, Teach one". HPB (Oxford). 2017;19(3):234-245.
16. de Rooij T, van Hilst J, Boerma D, et al. Impact of a nationwide training program in minimally invasive distal pancreatectomy (LAELAPS). Ann Surg. 2016;264(5):754-762.

17. Hogg ME, Tam V, Zenati M, et al. Mastery-based virtual reality robotic simulation curriculum: the first step toward operative robotic proficiency. J Surg Educ. 2017;74(3):477-485.

Publisher's Note Springer Nature remains neutral with regard to jurisdictional claims in published maps and institutional affiliations. 